\title{
Characterization of Ferrofluid-Based Stimuli-Responsive Elastomers
}

\begin{abstract}
Sandra de Pedro', Xavier Muñoz-Berbel', Rosalia Rodríguez-Rodríguez ${ }^{2,3}$, Jordi Sort', Jose A. Plaza ${ }^{1}$, Juergen Brugger ${ }^{5}$, Andreu Llobera ${ }^{1 \star}$ and Victor J. Cadarso ${ }^{6}$

${ }^{1}$ Esfera UAB, Institut de Microelectrònica de Barcelona IMB-CNM (CSIC), Bellaterra, Spain, ${ }^{2}$ Department of Pharmacology, University of Sevilla, Sevilla, Spain, ${ }^{3}$ Faculty of Medicine and Health Sciences, Basic Sciences Department, Universitat Internacional de Catalunya, Barcelona, Spain, ${ }^{4}$ Departament de Física, Institució Catalana de Recerca i Estudis Avançats (ICREA), Universitat Autònoma de Barcelona, Bellaterra, Spain, ${ }^{5}$ Microsystems Laboratory, École Polytechnique Fédérale de Lausanne (EPFL), Lausanne, Switzerland, ${ }^{6}$ Laboratory for Micro and Nanotechnology, Paul Scherrer Institut (PSI), Villigen, Switzerland
\end{abstract}

Stimuli-responsive materials undergo physicochemical and/or structural changes when a specific actuation is applied. They are heterogeneous composites, consisting of a non-responsive matrix where functionality is provided by the filler. Surprisingly, the synthesis of polydimethylsiloxane (PDMS)-based stimuli-responsive elastomers (SRE) has
OPEN ACCESS

Edited by:

Oscar Vazquez Mena,

University of California San Diego,

USA

Reviewed by:

Yi Chiu,

National Chiao Tung University,

Taiwan

Philipp Sebastian Schattling,

Aarhus University, Denmark

*Correspondence: Andreu Llobera

andreu.llobera@imb-cnm.csic.es

Specialty section:

This article was submitted to Micro- and

Nanoelectromechanical Systems, a section of the journal

Frontiers in Mechanical Engineering

Received: 05 July 2016

Accepted: 24 November 2016

Published: 12 December 2016

Citation:

de Pedro S, Muñoz-Berbel X, Rodríguez-Rodríguez R, Sort J, Plaza JA, Brugger J, Llobera A and Cadarso VJ (2016) Characterization

of Ferrofluid-Based StimuliResponsive Elastomers.

Front. Mech. Eng. 2:12. doi: 10.3389/fmech.2016.00012 seldomly been presented. Here, we present the structural, biological, optical, magnetic, and mechanical properties of several magnetic SRE (M-SRE) obtained by combining PDMS and isoparafin-based ferrofluid (FF). Independently of the FF concentration, results have shown a similar aggregation level, with the nanoparticles mostly isolated (>60\%). In addition to the superparamagnetic behavior, the samples show no cytotoxicity except the sample with the highest FF concentration. Spectral response shows FF concentrations where both optical readout and magnetic actuation can simultaneously be used. The Young's modulus increases with the FF concentration until the highest FF concentration is used. Our results demonstrate that PDMS can host up to 24.6\% FF (corresponding to $2.8 \%$ weight of $\mathrm{Fe}_{3} \mathrm{O}_{4}$ nanoparticles concentration). Such M-SRE are used to define microsystems - also called soft microsystems due to the use of soft materials as main mechanical structures. In that scenario, a large displacement for relatively low magnetic fields $(<0.3 \mathrm{~T})$ is achieved. The herein presented M-SRE characterization can be used for a large number of disciplines where magnetic actuation can be combined with optical detection, mechanical elements, and biological samples.

Keywords: stimuli-responsive materials, elastomers, ferrofluids

\section{INTRODUCTION}

Material engineering has in Nature a nearly infinite source of inspiration, stemming from materials with unique properties that result from billion years' evolution. Nature has defined molecular assemblies and interfaces suitable for specific function, and with the ability to adapt to different environments. Biomimetics or, more specifically, bioinspired functional materials (Darder et al., 2010) try to apply biological solutions to material science by imitating Nature. Examples of such are responsive biointerfaces (Yang et al., 2014), control of highly specific cell cultures (Fisher et al., 2010), mimicking muscles (Cheng et al., 2015), or as an active media for quantifying minute amounts of etiological agents (Darder et al., 2007). Among them, of especial relevance are those materials that 
undergo conformational or phase changes when external stimuli, such as changes in $\mathrm{pH}$ (Xiong et al., 2014) or electromagnetic fields (Shi et al., 2013), are applied. Such stimuli-responsive materials (SRM) (Roy et al., 2010) are heterogeneous composites, generally consisting of a filler - also called dopant or additive that provides the required functionality and a non-responsive host matrix, which confers mechanical robustness.

Clearly, the filler properties are crucial for any SRM, therefore they have to be selected in accordance to the actuation principle to be used. Fillers, mostly in the form of powders with submicrometer or nanometer particle sizes can provide the required properties to the SRM, including magnetic (Filipcsei et al., 2007), electric (Roy et al., 2010; Taleb et al., 2014), mechanical (Sagara and Kato, 2009; Shanmuganathan et al., 2010), or optic (Giammanco et al., 2015; Seo et al., 2015) properties. With regards to the matrix itself, organic and inorganic materials have already been used (Roy et al., 2010). Among them, polymers are gaining special attention since polymeric matrices can be functionalized in bulk, as opposite to inorganic materials, where only surface functionalization can be done. In addition, the polymeric 3D matrix assures the physical (and sometimes chemical) entrapment of the filler inside the polymer (Alves et al., 2010). Elastomers, and specially polydimethylsiloxane (PDMS), can easily be doped with magnetic particles, allowing the synthesis of a specific type of SRM - often called stimulus-sensitive or magneto-rheological elastomers, SRE or MRE, respectively. The selection of this elastomer is not at all arbitrary, since it exhibits outstanding properties such as: (i) low Young's modulus ( $Y$ ) (Carrillo et al., 2005); (ii) high transmittance in the UV-NIR range $(>80 \%)$ (Ibarlucea et al., 2010); (iii) biocompatibility (Munoz-Berbel et al., 2013); (iv) long-range thermo-chemical and structural stability (Xia and Whitesides, 1998); and (v) fabrication simplicity (Vila-Planas et al., 2011).

Among the myriad of possible applications, it is in the field of biomedicine where not only the PDMS but more importantly the SRMs (including SRE) have shown the highest potential (ViveroEscoto et al., 2010). Specifically, SRM for controlled release of theranostic agents (Wang et al., 2014), biosensing/diagnostics (Alves et al., 2010), or smart films/matrices for tissue engineering (Jeong and Gutowska, 2002; Fisher et al., 2010) have already been reported. In all of them, the ubiquitous requirement is that the SRM should also fulfill strict chemical, mechanical, biological, and operational assets: (i) chemically, materials with very low reactivity are preferred due to their inherent stability (Hotz et al., 2013), avoiding leaching of potentially toxic fillers (or subproducts); (ii) mechanically, SRMs should still have a low Young's modulus to more closely adapt to the working region and offer less mechanical resistance in response to an specific stimulus; (iii) biologically, SRM must not cause pro-inflammatory states or cysts that compromise the functionality of the device; and (iv) finally, systems should have small footprint, with contactless actuation and reversible (on/off transition). In this context, SRM suitable to change their shape under non-contact external physical stimulus, such as electromagnetic fields (Ahn et al., 2008) or optical means (Schattling et al., 2014), are preferred. Specifically, magnetically actuable SRM (M-SRM or, when using PDMS as matrix, M-SRE) have been reported in the literature (Zhang et al.,
2007), providing with actuation forces in the order of hundreds of millinewton using magnetic nanoparticles (MNPs) (Lu et al., 2007) additive to the PDMS matrices (Khoo and Liu, 2001).

M-SRE have shown large shape tunability and changes in its mechanical properties as a function of the dispersion, distribution, morphology, and loading of the MNPs (Pankhurst et al., 2003; Han et al., 2013). Nevertheless, the mixture of solid (powder) magnetic additives with PDMS to obtain such M-SRE is suboptimal, since this approach is prone to the formation of aggregates. These heterogeneities induce non-homogeneous functionality of the material, which results in poor performance (i.e., uneven physicochemical properties). Moreover, aggregates may drastically modify the optical properties of the obtained material, increasing its surface roughness, changing its surface properties, and hindering the bonding process, hence hampering the systematic use of this material.

An alternative to the aforementioned is to disperse MNPs in carrier liquids. Such stable colloidal form is generally known as ferrofluid (FF) (Pileni, 2001). Among the different commercially available FFs, those containing magnetite $\left(\mathrm{Fe}_{3} \mathrm{O}_{4}\right)$ nanoparticles immersed in oil as carrier liquid, with diameter ranging between 3 and $15 \mathrm{~nm}$ and presenting either superparamagnetic or singledomain magnetic behavior, are preferred. Among their properties, such FFs are non-toxic and non-carcinogenic and they exhibit high resistance to oxidation, mainly because of their polymeric capping. Currently, those materials have been used in biological applications, including cellular therapy (Lubbe et al., 2001), drug delivery (Lubbe et al., 2001), magnetic resonance imagining (Hong et al., 2008), and hyperthermia (Jordan et al., 1997).

Even being an extremely promising material, the physicochemical and biological properties of FF-based M-SREs have seldomly been jointly reported (Stuart et al., 2010). In this work, we aim to tackle this issue by characterizing, using various complementary techniques, M-SREs obtained from PDMS as host matrix and isoparafin-based FF containing $\mathrm{Fe}_{3} \mathrm{O}_{4}$ nanoparticles as additive. Different properties of the M-SRE as a function of FF concentration are thoroughly investigated. First, the internal structure of the M-SRE (i.e., the particle size, the eventual formation of aggregates, and the structural homogeneity) has been studied by transmission electron microscopy (TEM) analysis. Then, a biocompatibility study of the M-SRE has been carried out to determine if the M-SRE is suitable for cell culture, and how the cell proliferation is affected by the FF concentration. Following, the M-SRE has been both magnetic and optically characterized in bulk and mechanically studied by defining M-SRE cantilevers. As a final example of application, the M-SRE has been used for defining magnetically actuable soft microsystems and its deflection as a function of the applied magnetic field has been measured.

\section{RESULTS AND DISCUSSION}

\section{Material Preparation}

M-SRE was obtained by mixing PDMS pre-polymer (1:10 in volume curing agent/base elastomer) and six different FF volume ratios $\left(\mathrm{S}_{0}=0 \% ; \mathrm{S}_{1}=1.7 \% ; \mathrm{S}_{2}=3.5 \% ; \mathrm{S}_{3}=9.8 \% ; \mathrm{S}_{4}=15.4 \%\right.$; $\mathrm{S}_{5}=20.3 \%$; and $\mathrm{S}_{6}=24.6 \%$ ), corresponding to $\mathrm{Fe}_{3} \mathrm{O}_{4}$ nanoparticles' 
weight concentrations of $\mathrm{S}_{0}=0 \% ; \mathrm{S}_{1}=0.2 \% ; \mathrm{S}_{2}=0.4 \% ; \mathrm{S}_{3}=1.1 \%$; $\mathrm{S}_{4}=1.7 \% ; \mathrm{S}_{5}=2.3 \%$; and $\mathrm{S}_{6}=2.8 \%$. After homogeneous dispersion, the non-polymerized material was degassed to remove air bubbles and baked so as to achieve 3D polymeric cross-linking.

\section{Sample Patterning}

The structural homogeneity, biocompatibility, optical transmittance, and magnetization were studied in bulk using $2 \mathrm{~cm} \times 2 \mathrm{~cm}$ slab samples and having a thickness of $250 \mu \mathrm{m}$. The M-SREs Young's modulus has been obtained by fabricating the cantilevers shown in Figure 1A with dimensions $500 \mu \mathrm{m}$ (width, $w$ ), 4,400 $\mu \mathrm{m}$ (length, $l$ ), and $250 \mu \mathrm{m}$ (thickness, $h$ ) and by measuring the maximum deflection when $1 \mathrm{~g}$ acceleration was applied. Figure 1B shows the fabricated cantilever using $\mathrm{S}_{5}$. The final soft microsystem was focused toward enhancing its magneto-mechanical response. To this effect, a rectangular seismic mass (with dimensions $w_{\mathrm{m}}, l_{\mathrm{m}}$, and $h_{\mathrm{m}}$, in micrometers being of 1,600,2,500, and 250) was included at the cantilever tip, as shown in Figure 1C (design) and Figure 1D (also fabricated using $\mathrm{S}_{5}$ ).

\section{Structural Properties}

One of the most important aspects on synthetizing SRE is to set the filler concentration upper threshold that the polymeric matrix can host, since incomplete polymerization may lead to fragile, brittle, or materials with non-homogeneous response that may leach the filler and therefore have a time-dependent actuation. For thermocurable materials - such as the family of M-SREs here presented - the curing time is indicative of how the filler affects the 3D cross-linking of the polymeric matrix. In this context, the reference was taken from the curing step for the non-doped material $\left(\mathrm{S}_{0}\right.$, with $\left.0 \mathrm{wt} \% \mathrm{FF}\right)$, which was of $20 \mathrm{~min}$ at $80^{\circ} \mathrm{C}$. Identical curing step was required for samples $S_{1}-S_{3}$, indicative that the filler, in the concentrations used for these specific M-SRE, did not affect the formation of the $3 \mathrm{D}$ polymeric matrix at these concentrations. For samples $S_{4}$ and $S_{5}$, the curing time was increased to $25 \mathrm{~min}$ and for sample $S_{6}$ up to $35 \mathrm{~min}$. Although it was possible to cure this last sample, such an increase of the curing time was highly significant, and variations on the polymer structural properties were to be expected. For this reason, this FF concentration was considered to be the upper threshold.
A

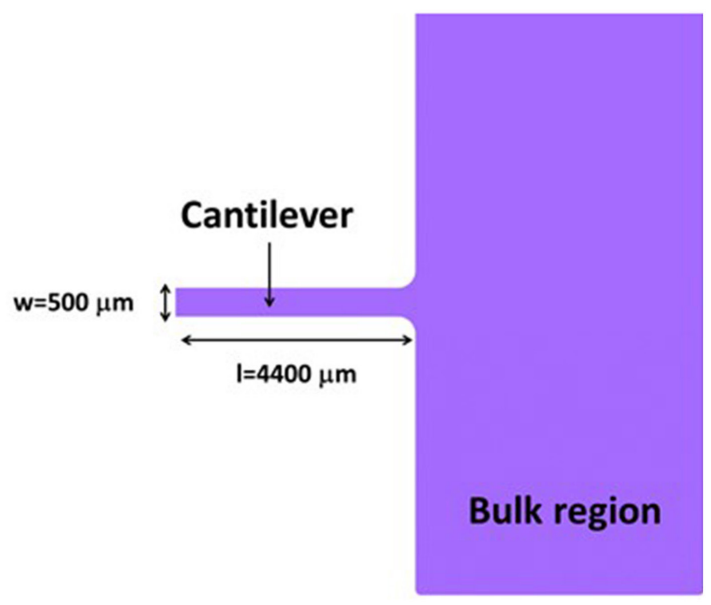

C

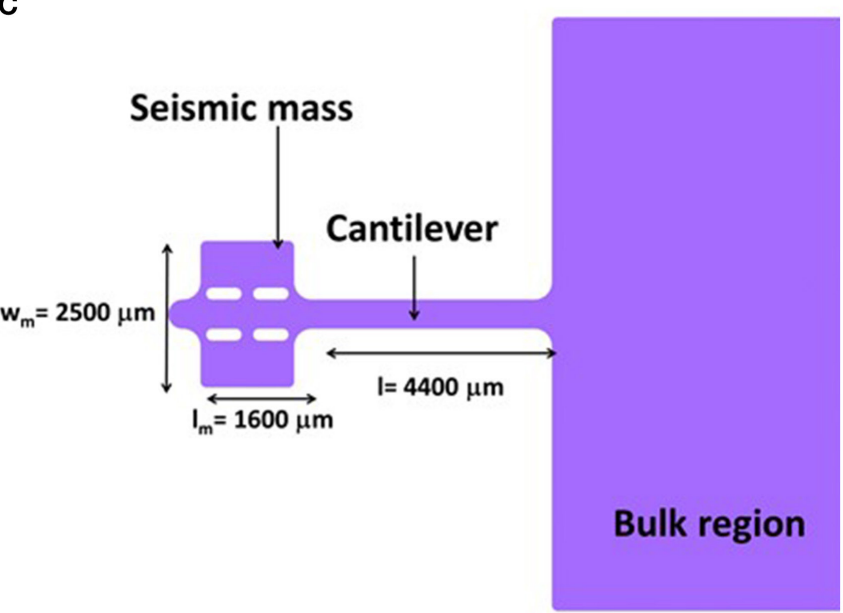

B

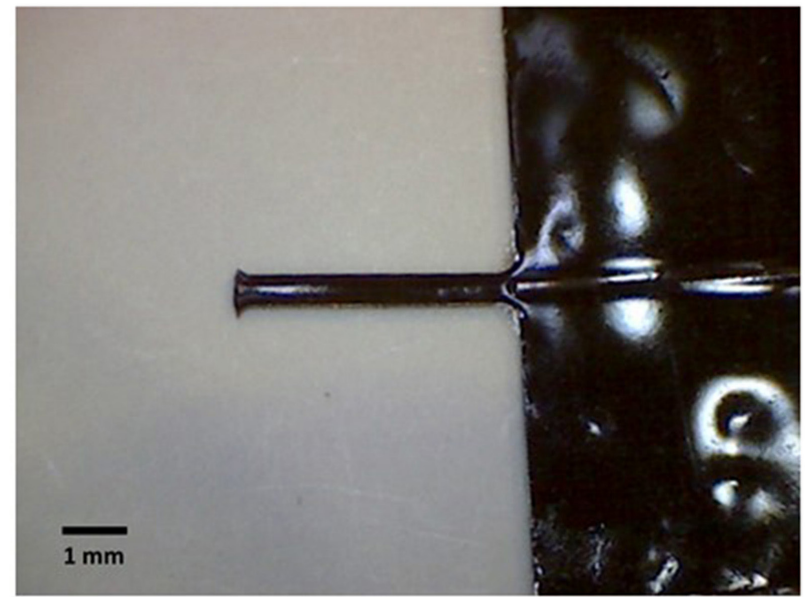

D

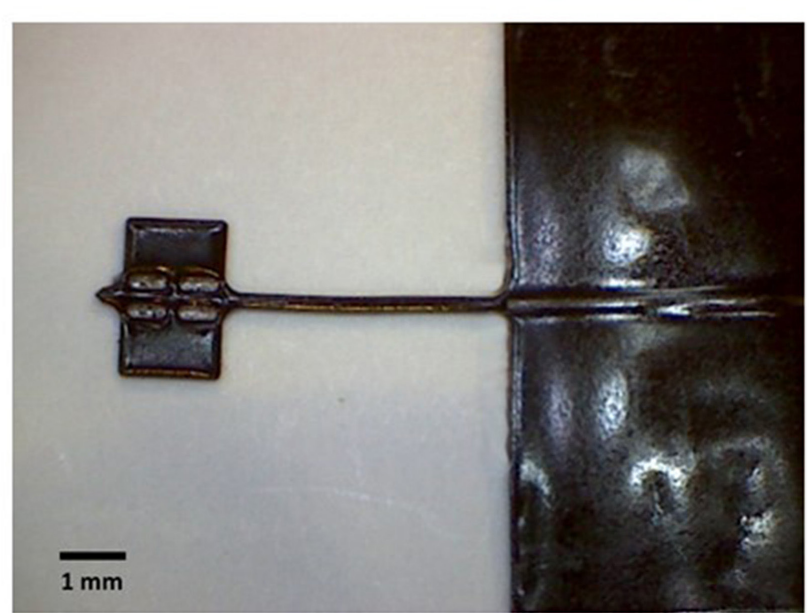

FIGURE 1 | (A) Design and (B) fabricated cantilevers using M-SRE for measurement of the Young's modulus using $S_{5}$ M-SRE. (C) Design and (D) fabricated soft microsystems using the same material. In both cases, the thickness of the structures was of $250 \mu \mathrm{m}$. 
In parallel, slab samples with low $\left(\mathrm{S}_{2}\right)$ and high $\left(\mathrm{S}_{6}\right)$ FF concentration were used for the image analysis to calculate the average particle diameter, taking into account that the MNP included in the FF have the magnetic core and a polymeric capping. Histograms illustrating nanoparticle size distribution $\left(D_{\mathrm{TEM}}\right)$ are presented in Figures $\mathbf{2 A , B}$, together with representative TEM images (insets in both figures). The deconvolution of such histograms in Gaussian peaks can also be seen in Figure 2, and more detailed information is provided in Table 1. For sample $S_{2}$ (Figure 2A), a predominant peak centered at a particle size of $8.9 \pm 0.1 \mathrm{~nm}$ was obtained, which could be associated to nonaggregated MNP. A secondary peak was also noticeable located at a particle size of $15.5 \pm 0.5 \mathrm{~nm}$. With an area of only $11 \%$ as compared to the predominant peak, this second Gaussian peak is due to aggregates of two MNPs. Figure 2B shows that the histogram for sample $S_{6}$ could be deconvoluted into three main peaks, corresponding to single MNPs $(8.2 \pm 0.1 \mathrm{~nm})$, two MNPs' aggregate $(13.2 \pm 0.1 \mathrm{~nm})$, and three MNPs' aggregate $(19.0 \pm 0.6 \mathrm{~nm})$. As compared to the predominant peak of this sample, peaks \#2 and \#3 had a relative area of 44 and 13\%, respectively. These data suggested that the increase of MNPs caused a significant increase of small aggregates - formed by two or three MNPs - which do not hamper the overall behavior of the M-SRE since their distribution is homogeneous in the material, as it can be seen at the insets of Figure 2. From these data, it can be confirmed that the PDMS matrix can host up to four times more FF volumes than those previously presented (Pirmoradi et al., 2010; Balasoiu et al., 2011) without having large aggregates. In turn, this higher FF concentration will assure a higher actuation in response to a magnetic stimulus, as it will be discussed in the following sections.

\section{Biocompatibility}

The development of lab on chip systems and MEMS has been strongly linked to biomedical applications, becoming a major application field for these architectures. Lab on a chip systems for biomedicine should guarantee the integrity and stability of the sample under study, that being biological fluids, cells, or tissues, among others. The requirements for materials and systems applied to biomedicine are thus very strict, being biocompatibility one of the most important to be determined.
Based on the latter, the biocompatibility of the proposed material was evaluated using vascular smooth muscle cells (VSMC) from rat aorta as model eukaryotic cells, considering their relevance in cardiovascular pharmacology. Figure 3 shows the cell viability for M-SRE samples (from $\mathrm{S}_{0}$ to $\mathrm{S}_{6}$ ). Viability tests were performed using the colorimetric MTT (3-(4,5-dimethylthiazol-2-yl)-2,5-diphenyltetrazolium bromide) assay (Mosmann, 1983) After pre-attachment of the VSMC in the well, M-SRE samples with a total volume of $40 \mathrm{~mm}^{3}$ were introduced in each well, and the cell viability was measured after $24 \mathrm{~h}$ of incubation with the MTT.

TABLE 1 | Summary of the deconvoluted peaks for studying the particle size distribution of samples $S_{2}$ and $S_{6}$.

\begin{tabular}{lccllcc}
\hline & \multicolumn{2}{c}{$\mathbf{S}_{\mathbf{2}}$} & & \multicolumn{3}{c}{$\mathbf{S}_{6}$} \\
\cline { 2 - 3 } \cline { 7 - 7 } \cline { 5 - 7 } & Peak \#1 & Peak \#2 & & Peak \#1 & Peak \#2 & Peak \#3 \\
\hline Center max (nm) & $8.9 \pm 0.1$ & $15.5 \pm 0.5$ & & $8.2 \pm 0.1$ & $13.2 \pm 0.1$ & $19.0 \pm 0.6$ \\
Area (frequency-nm) & $88 \pm 2$ & $10 \pm 2$ & & $62 \pm 3$ & $27 \pm 4$ & $9 \pm 2$ \\
FWHM (nm) & $6.4 \pm 0.2$ & $5.2 \pm 0.9$ & & $4.9 \pm 0.2$ & $4.1 \pm 0.5$ & $5 \pm 1$
\end{tabular}

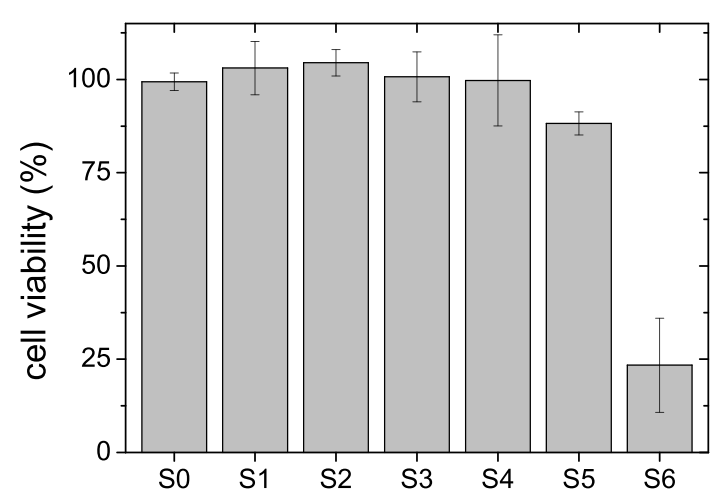

FIGURE 3 | Measured cell viability (\%) obtained from vascular smooth muscle cells using the colorimetric MTT (3-(4,5-dimethylthiazol-2-yl)2,5-diphenyltetrazolium bromide) assay when exposed to different amounts $\mathbf{M}-\mathbf{S R E}$ samples (from $\mathbf{S}_{0}$ to $\mathbf{S}_{6}$ ). Results are expressed as the mean $\pm \operatorname{SEM}(n=3-6) .{ }^{*} P<0.05 ;{ }^{\star \star \star} P<0.001$ vs. So.
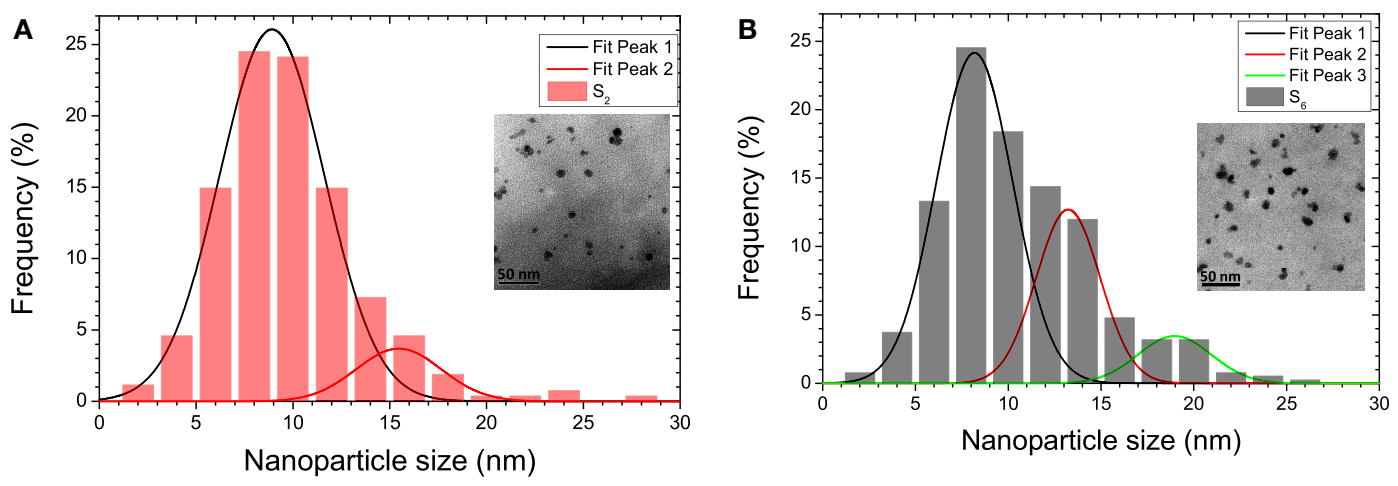

FIGURE 2 | Nanoparticle size distribution by transmission electron microscopy (TEM) image analysis and Gaussian fitting for (A) $3.5 \%$ ( $\left.\mathrm{S}_{2}\right)$ and $(\mathrm{B})$ $\mathbf{2 4 . 6} \%\left(\mathbf{S}_{6}\right) \mathbf{M}$-SRE. Inset in each figure corresponds to TEM images. 
Viability was determined as a percentage in relation to control samples, i.e., cells proliferation in a well without M-SRE. Between three and six replicates were done for each M-SRE for statistical validation. $S_{0}-S_{4}$ samples showed values similar to $100 \%$ viability. On the contrary, sample $\mathrm{S}_{5}$ and particularly $\mathrm{S}_{6}$ showed toxicity. In sample $\mathrm{S}_{4}$, the $\%$ cell viability was of $99 \pm 12 \%$. This magnitude decreased until $88 \pm 3 \%$ for $S_{5}$ and $23 \pm 13 \%$ for $S_{6}$. Therefore, it can be concluded that $\mathrm{S}_{0}-\mathrm{S}_{4}$ samples are suitable for biological applications.

\section{Magnetization}

Figure 4 shows the experimentally measured magnetization as a function of the applied magnetic field, $\mu_{0} H$, for slab samples $S_{1}-S_{6}$ at $300 \mathrm{~K}$. None of them shows either coercitivity or remanence, which is indicative of their superparamagnetic behavior. Actually, this is in agreement with the superparamagnetic nature of the $\mathrm{Fe}_{3} \mathrm{O}_{4}$ nanoparticles at room temperature (Ivaneyko et al., 2012). On the same figure, the saturation magnetization $\left(m_{\mathrm{s}}\right)$ (understood as the total magnetization corresponding to an applied field of 1.2 T) for each sample is included.

According to the superparamagnetic theory (Cullity, 1974), the magnetic particle size can be calculated from the true magnetic moment of each particle $(\mu)$ using the Langevin Eq. 1, which was used for fitting the magnetization curves shown in Figure 4 .

$$
m=m_{\mathrm{S}}\left[\operatorname{coth}\left(\frac{\mu H}{k_{\mathrm{B}} T}\right)-\frac{k_{\mathrm{B}} T}{\mu H}\right],
$$

where $k_{\mathrm{B}}$ is the Boltzmann constant, $T$ is the absolute temperature, $m_{\mathrm{S}}$ is the saturation magnetization, $H$ is the magnetic field, and $\mu$ is the true magnetic moment, which is defined as

$$
\mu=m_{\mathrm{S}} \pi D_{\mathrm{L}}^{3} / 6
$$

being $D_{\mathrm{L}}$ is the magnetic particle size.

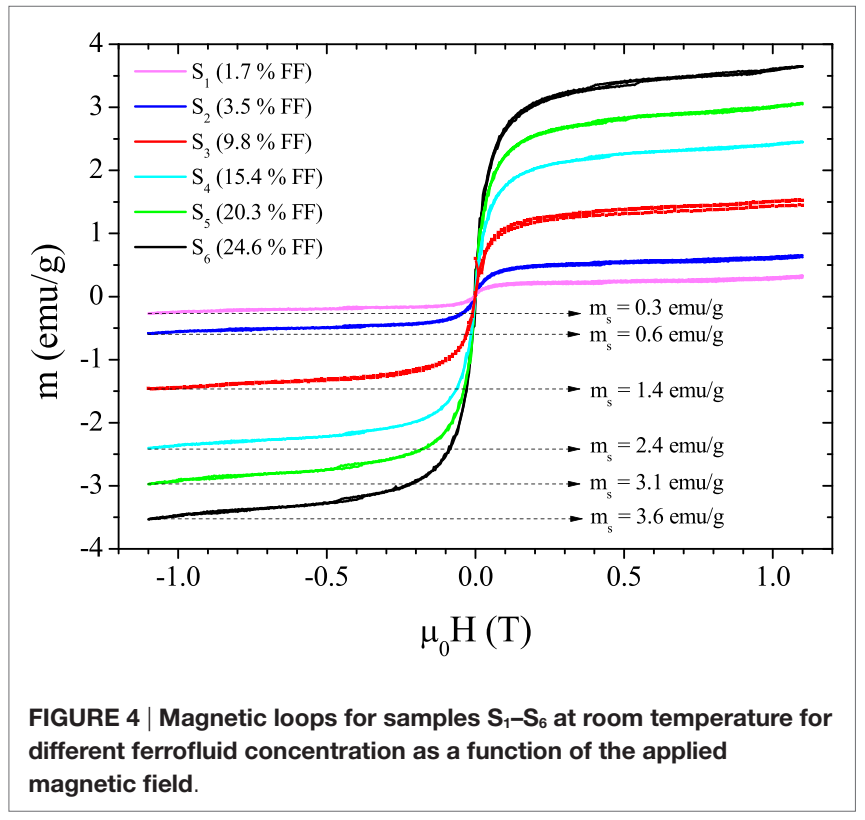

The obtained $D_{\mathrm{L}}$ values were compared with the values from TEM observations, i.e., those corresponding to the main peak i.e., without aggregates $-\left(D_{\mathrm{TEM}}\right)$. The results are summarized in Table 2. Differences between $D_{\mathrm{L}}$ and $D_{\text {TEM }}$ were due to the fact that magnetic measurements did not take into account the presence of the capping MNPs layer. The particle size was recalculated including the thickness of the capping, which is $a_{0}=0.83 \mathrm{~nm}$ for magnetite (Kaiser and Miskolcz, 1970). Introducing this correction into the Langevin function leaded to a modified magnetic particle size $D_{\mathrm{L}}^{\prime}=D_{\mathrm{L}}+a_{0}$. Once this aspect was considered, a good agreement was obtained then between the $D_{\mathrm{L}}^{\prime}$ value from Eq. 2 and the TEM measurements (Table 2). On the basis of such results, it can be confirmed that the small aggregates formed in the M-SRE - comprised of two or three MNPs - and its homogeneous distribution in the sample do not cause a significant change of its magnetic properties, even in the samples with the highest FF concentration.

\section{Young's Modulus of M-SRE}

Materials with Young's modulus in the order of kilopascal-megapascal are very attractive for life science applications since they present similar stiffness as biological entities such as arteries (Rodriguez-Rodriguez et al., 2014). In that context, since the main aim of this paper is to develop soft microsystems, it becomes crucial to experimentally measure the effect of the FF on the Young's modulus of the SRE. By measuring the maximum deflection $\left(y_{\max }\right)$ of the cantilevers upright and upside down, the $Y_{\mathrm{M} \text {-SRE }}$ was experimentally obtained by subtracting the initial offset of the cantilever and using Eq. 3.

$$
Y_{\text {M-SRE }}=\frac{3 \rho g l^{4}}{2 y_{\max } h^{2}},
$$

where $\rho$ is the M-SRE density, $g$ the acceleration of the gravitational field, and $l$ and $h$ are the length and thickness of the cantilever, respectively.

Figure 5 shows the experimental $Y_{\mathrm{M}-\mathrm{SRE}}$ values obtained. From an initial value close to $200 \mathrm{kPa}$ for samples $\mathrm{S}_{0}-\mathrm{S}_{2}, Y_{\mathrm{M} \text {-SRE }}$ rapidly increases with the FF concentration until it reaches $20.3 \%$ volume FF. The observed increase of $Y_{\mathrm{M} \text {-SRE }}$ is quadratic with the FF concentration for samples $S_{1}-S_{5}$, as predicted by Bergstrom and Boyce (1999). Conversely, $S_{6}$ does not follow such tendency. The reason for this could be the above described incomplete formation of the $3 \mathrm{D}$ polymeric network due to the FF oversaturation, which again seems to confirm that the concentration limit for this specific FF in PDMS is obtained with $\mathrm{S}_{5}$ sample.

As compared to the previously published results, the values here obtained are similar to those provided by the manufacturer datasheet $\left(Y_{\mathrm{PDMS}}=300-800 \mathrm{kPa}\right)$, even though other researchers

TABLE 2 | Values of nanoparticle size obtaining from TEM study $\left(D_{\text {TEM }}\right)$, from Eq. $2\left(D_{L}\right)$, and by including the capping thickness $\left(D_{L}^{\prime}\right)$ for samples $\mathrm{S}_{2}$ and $\mathrm{S}_{6}$.

\begin{tabular}{lccc}
\hline Sample & $\boldsymbol{D}_{\mathbf{L}}(\mathbf{n m})$ & $\boldsymbol{D}_{\text {TEM }}(\mathbf{n m})$ & $\boldsymbol{D}_{\mathbf{L}}^{\prime}(\mathbf{n m})$ \\
\hline $\mathrm{S}_{2}$ & $6 \pm 1$ & $8.9 \pm 0.1$ & $8 \pm 1$ \\
$\mathrm{~S}_{6}$ & $4 \pm 1$ & $8.2 \pm 0.1$ & $6 \pm 1$
\end{tabular}




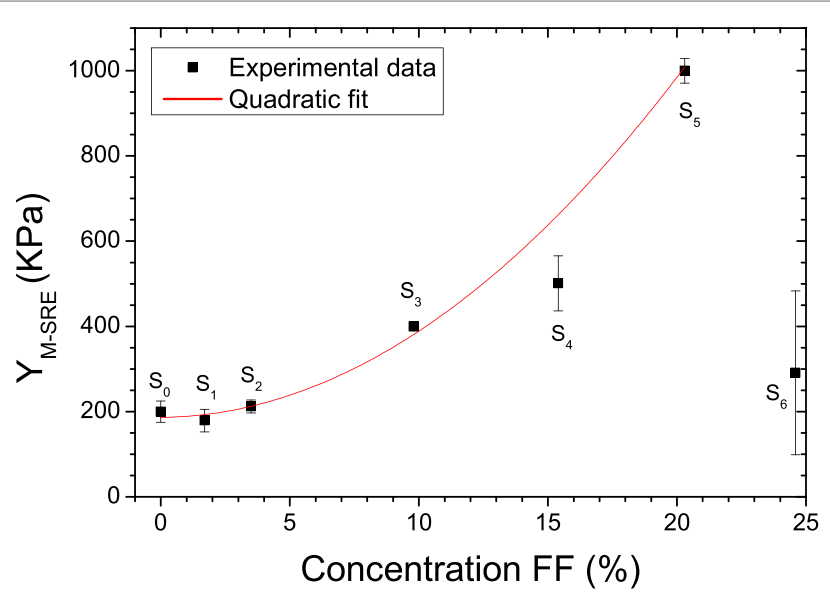

FIGURE 5 | Dependence of the Young's modulus of the M-SRE ( $\left.Y_{\mathrm{M}-\mathrm{SRE}}\right)$ as a function of the ferrofluid concentration in the PDMS. An increase of $Y_{M-S R E}$ is observed until it reaches the maximum value corresponding to $20.3 \%$, beyond which a drop of $Y_{M-S R E}$ is observed.

have shown that $Y_{\text {PDMS }}$ can be in the range of the megapascal (Johnston et al., 2014). As a rule, it has been demonstrated that $Y_{\mathrm{PDMS}}$ strongly varies on both the curing agent/base elastomer ratio, temperature, baking time, and baking conditions (Fuard et al., 2008; Johnston et al., 2014). Specifically, in Brau et al. (2011) and Terwagne et al. (2014), the Young's modulus of PDMS is shown to vary between 10 and $1,000 \mathrm{kPa}$ depending of the aforementioned technological conditions. In that sense, the values obtained here are in agreement with those previously published.

\section{Transmittance in the VIS-NIR}

The optical properties of a polymeric matrix may strongly vary when an additive is included. When synthetizing the M-SRE, not only the particle size, distribution, shape, or capping of the FF but also the optical and chemical properties of the liquid carrier may potentially modify the optical transmittance of the M-SRE. This change is crucial if the idea is to use this material for defining magnetically actuable elements to be implemented in photonic lab on a chip systems (PhLoC) (Vila-Planas et al., 2011), for example, valves or pumps, where the whole spectral response of a given analyte is acquired in real time. Before such integration can be even considered, it becomes necessary to determine the effect of the FF concentration on the PDMS transmittance. Results presented in Figure $\mathbf{6}$ shows the transmittance for slab samples $\mathrm{S}_{0}$ to $S_{6}$ in a wavelength range between 400 and $900 \mathrm{~nm}$. In all cases, a propagation distance of the light in material of $250 \mu \mathrm{m}$ has been used. As expected, the increase of the FF concentration resulted in a sharp decrease of the transmittance due to scattering centers and specific optical absorption from FF. Samples $S_{1}-S_{2}$ presented minimum transmittance (at a wavelength of $400 \mathrm{~nm}$ ) of 59 and $46 \%$, respectively. Therefore, these M-SRE can be considered suitable for developing PhLoC with magnetic actuation. Sample $\mathrm{S}_{3}$ has a transmittance at the same wavelength of $30 \%$, which may still be valid for PhLoC with short optical paths. Finally, samples

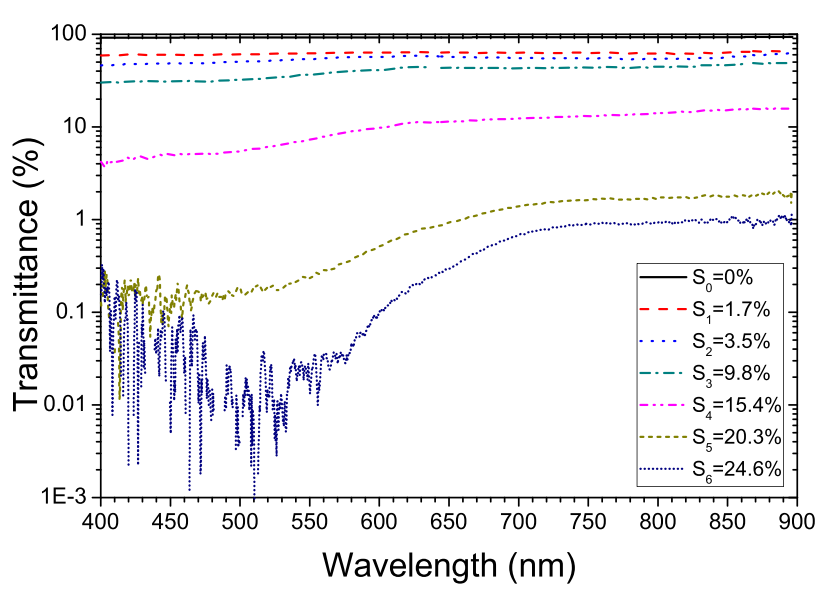

FIGURE 6 | Transmittance as a function of wavelength for slab samples with a thickness of $250 \mu \mathrm{m}$ of PDMS $\left(\mathrm{S}_{0}\right)$ and the M-SREs $\left(\mathbf{S}_{1}-\mathrm{S}_{6}\right)$.

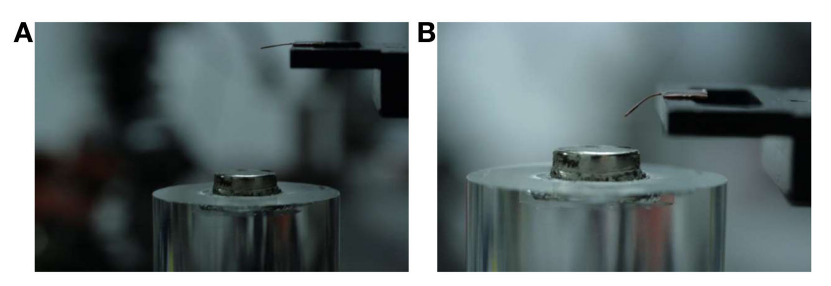

FIGURE 7 | Cantilever defined with sample $\mathrm{S}_{4}$ positioned over a permanent magnet, showing (A) no deflection for weak magnetic fields (i.e., more than $3 \mathrm{~cm}$ between the cantilever and the permanent magnet) and (B) deflection under comparatively larger magnetic fields.

with larger FF concentration $\left(\mathrm{S}_{4}-\mathrm{S}_{6}\right)$ can be considered as opaque in the VIS-NIR range and, even though they will have a higher displacement upon magnetic actuation, they cannot be considered as valid M-SRE to implement PhLoCs or, more generally to develop elastomer-based systems where visual inspection or optical detection is required.

\section{Characterization of the Soft Microsystems Fabricated with the M-SRE}

Once the M-SRE was characterized, the next step was to show the applicability of this material. Specifically, six different soft microsystems were fabricated - one for each FF concentration and showed deflection in the order of hundreds of micrometer when an external magnetic field is applied. Figure 7 presents a cantilever fabricated with sample $\mathrm{S}_{4}$ showing no actuation for weak applied magnetic fields (Figure 7A) and large displacements when larger magnetic fields are applied (Figure 7B).

Quantification of this deflection as a function of the applied magnetic field was measured, and it is shown in Figure 8. As it can be seen, deflection of the soft microsystem shows the expected increase in magnitude with $\mu_{0} H$ for all M-SREs under study. For a specific deflection target, higher $\mu_{0} H$ is required for 
samples with low FF concentration. As an example, for reaching a deflection of $500 \mu \mathrm{m}$, an applied magnetic field of $0.33 \mathrm{~T}$ is needed for $S_{1}$, whereas it can be decreased to $0.2 \mathrm{~T}$ for $S_{5}$. Finally, the mechanical sensitivity of the M-SRE with larger FF content is the highest. This means that larger deflections can be obtained but, equally important, small changes of this applied magnetic field will cause very significant variances in the resulting deflection. Therefore, for robust microsystems similar to these here presented, the optimal alternative is the $S_{3}$ material, where the deflection can accurately be controlled with the applied magnetic field. Microsystems done with $\mathrm{S}_{4}$ and $\mathrm{S}_{5}$ are still valid for ON/OFF actuation, such as microvalves. For quantitative actuation, they could be used by making the microsystems stiffer, i.e., increase the thickness or decrease its length.

To summarize, the different studied properties of the here discussed M-SRE in view of their application to implement magnetic soft microsystem are shown in Table 3. The selection of the given FF concentration strongly depends on the application. Low FF content materials $\left(\mathrm{S}_{1}-\mathrm{S}_{2}\right)$ are suitable for photonic and biological applications (higher optical transmittance and biocompatibility) even when the required applied magnetic field so as to achieve actuation is the highest. $S_{4}$ and $S_{5}$ are opaque materials that present a significant increase of its Young's modulus. The high FF concentration allows the largest deflection for relatively small

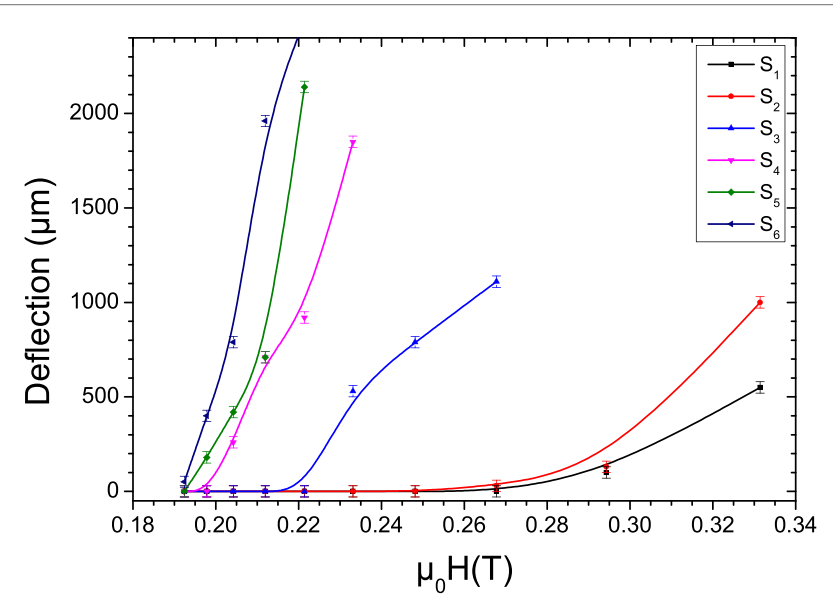

FIGURE 8 | Measured deflections as a function of the magnetic actuation for cantilevers fabricated with M-SREs $\mathbf{S}_{1}-\mathbf{S}_{6}$. applied magnetic fields, and therefore they are may be suitable for ON/OFF applications. A strategy if strong magnetic actuation is required in combination with photonics readout is to define the system in such a way that $S_{4}$ or $S_{5}$ M-SRE are only defined in specific regions (de Pedro et al., 2014), while the rest of the PhLoC is defined using $S_{0}$ (non-doped). In this approach, the advantages of both the types of materials could be merged in a single system, thus improving its performance. Measurements done with material $\mathrm{S}_{6}$ seem to be indicative that the polymeric $3 \mathrm{D}$ cross-linking is distorted due to FF oversaturation. Finally, $\mathrm{S}_{3}$ can be considered to be the optimal material, where biocompatibility, low $Y_{\mathrm{M}-\mathrm{SRE}}$, acceptable transmittance (for short optical paths) and, when used for microsystems, robust and controllable deflections, is achieved.

\section{CONCLUSION}

A study of the M-SREs obtained by incorporating different concentrations of FF based on $\mathrm{Fe}_{3} \mathrm{O}_{4}$ nanoparticles into a PDMS matrix has been presented, as well as its applicability to implement magnetic soft microsystems. Particle size distributions in the M-SRE have been studied using TEM. Histograms have shown that, even for the highest concentration of FF $(24.6 \%$, corresponding to $2.8 \%$ weight of $\mathrm{Fe}_{3} \mathrm{O}_{4}$ ), the main peak corresponds to single nanoparticles, with size of $8.2 \pm 0.1 \mathrm{~nm}$. This value is in agreement with the magnetic study of the M-SRE. Additionally, the magnetization as a function of the applied magnetic field for samples $S_{1}-S_{6}$ shows neither coercitivity nor remanence, in agreement with the superparamagnetic nature of the used FF. The study of the transmittance in the visible range as a function of the FF concentration has shown three different categories: $S_{1}$ and $S_{2}$ have transmittance higher than $50 \%$. $S_{3}$, with a transparency of $32 \%$, could still be valid for applications having short optical paths. Finally, samples $S_{4}-S_{6}$ are optically opaque. From a mechanical point of view, the $Y_{\mathrm{M} \text {-SRE }}$ shows the expected quadratic dependence on the FF concentration, except for the drastic decrease at the highest FF concentration, which has been associated to incomplete 3D polymer cross-link. Biocompatibility tests have shown that the M-SRE presented in this work are biocompatible in most of the samples, and only those with a highest FF concentration presents cytotoxicity. As applicability demonstrator, soft microsystems have been fabricated with these M-SRE using soft lithography, obtaining deflections of hundreds of micrometers as a function of $\mu_{0} H$ and of the specific FF concentration.

TABLE 3 | Summary of the parameters obtained in the characterization of M-SRE.

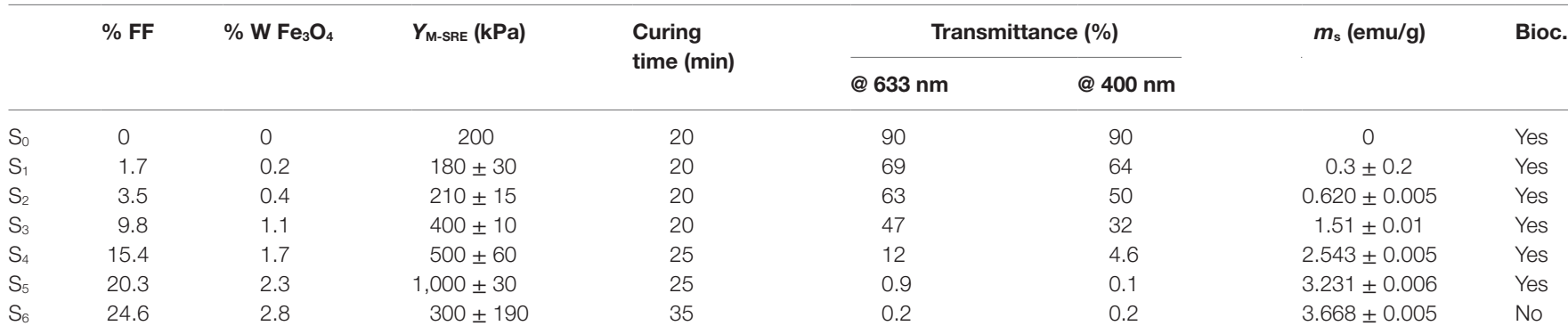




\section{EXPERIMENTAL SECTION}

\section{Reagents}

The negative-tone epoxy-based polymer SU-8 50 and its developer propylene glycol methyl ether acetate (PGMEA) (both from MicroChem, Corp., USA) were used following the datasheet specifications. PDMS elastomer (Sylgard Silicon Elastomer 184, Dow Corning Corp., USA) was prepared in accordance to the datasheet. FF was used as provided by the manufacturer (Liquids Research Limited, North Wales). The FF presents a uniform size distribution and low $\mathrm{Fe}_{3} \mathrm{O}_{4}$ particle agglomeration $(<20 \mathrm{~nm})$ dispersed into isoparafin with $10 \mathrm{~nm}$ as the mean particle diameter and a saturation magnetization of $400 \mathrm{G}$.

\section{Synthesis of Magnetic Stimuli-Responsive Elastomer (M-SRE)}

M-SRE was synthesized by mixing PDMS and FF. First, the PDMS pre-polymer was obtained in a 1:10 (v:v) curing agent/ base elastomer as available from the manufacturer. Just afterward, different FF volumes were included $(0 ; 1.7 ; 3.5 ; 9.8$; $15.4 ; 20.3$; and $24.6 \%$ in volume) and manually stirred until homogeneous dispersion is achieved. This mixture was then degassed to remove air bubbles. The resulting M-SREs were labeled as $S_{i}(i=0,1, \ldots, 6$; corresponding to the increasing FF concentrations).

\section{Fabrication}

The slab samples, cantilevers, and soft microsystems were fabricated with soft lithography technology (Vila-Planas et al., 2011). The process started using a soda-lime wafer with a thickness of $700 \mu \mathrm{m}$, onto which a $250 \mu \mathrm{m} \mathrm{SU}-850$ layer was spun. After 3-h baking at $95^{\circ} \mathrm{C}$, the wafer was exposed to UV light with a dose of $350 \mathrm{~mJ} / \mathrm{cm}^{2}$ using the appropriate mask. The post exposure bake for $20 \mathrm{~min}$ at $95^{\circ} \mathrm{C}$ and the development using PGMEA finished the master fabrication. M-SRE pre-polymer obtained as above mentioned was poured onto the SU-8 master, avoiding overflow. A second degasification was done so as to remove air bubbles that may be trapped in the microstructures. Then, they were baked for completing polymerization. Just afterward, the slab samples, cantilevers, and soft microsystems, having a thickness of $250 \mu \mathrm{m}$, were peeled off from the master with the help of a tweezer and positioned over a glass substrate for handling easiness.

\section{Structural Analysis}

Structural homogeneity was determined using images from TEM, which were obtained by the system JEOL 2011, Jeol Ltd., USA. The distribution and size of the $\mathrm{Fe}_{3} \mathrm{O}_{4}$ nanoparticles included in the M-SRE was analyzed. From these images, 10 fields containing each of them between 20 and 100 MNP depending on the sample were analyzed by image software (ImageJ, USA).

\section{Biocompatibility}

Polydimethylsiloxane is known to be a compatible material with low or no toxicity (Wu et al., 2010), making it suitable to be used with samples of biological origin (cells, enzymes, bacteria, etc.). The study of biocompatibility of the developed M-SRE was carried out for verifying whether the inclusion of FF in the PDMS altered the toxicity of the polymeric matrix. Here, samples from $\mathrm{S}_{0}$ to $\mathrm{S}_{6}$ were subjected to cytotoxicity study. The cytotoxicity was determined using VSMC cultured from rat aorta in combination with the magnetic samples (introduced into the wells where the experiment was conducted) by using the colorimetric MTT (3-(4,5-dimethylthiazol-2-yl)-2,5-diphenyltetrazolium bromide) assay (Mosmann, 1983). This technique is based on the ability of viable cells to transform the MTT salt into formazan dyes. After a 24-h VSMC incubation period, wells were washed with phosphate-buffered solution, and the MTT solution $(1 \mathrm{mg} / \mathrm{ml})$ was added and incubated for $4 \mathrm{~h}$. The purple formazan generated by viable cells was solubilized with $20 \%$ sodium dodecyl sulfate in $0.02 \mathrm{M} \mathrm{HCl}$ and incubated for $10 \mathrm{~h}$ at $37^{\circ} \mathrm{C}$. The optical density of each well was determined at $540 \mathrm{~nm}$ in a microplate spectrophotometer reader $\left(\right.$ BioTek $^{\circledR}$ Synergy HT). Cell viability was expressed as percentage in relation to controls (non-treated cells).

\section{Magnetization}

In order to determine the magnetic properties of the M-SREs, a vibrating sample magnetometer (VSM) (ADE model 10 VSM Oxford Instruments, UK) was used for obtaining the magnetization of the slab samples $S_{1}-S_{6}$ at $300 \mathrm{~K}$. Moreover, from this study the nanoparticle size was determined and compared with the results obtained from TEM.

Cantilevers and soft microsystem were fabricated using samples $S_{1}-S_{6}$. These structures were magnetically characterized by placing them on a positioning stage over a permanent magnet ( $0.45 \mathrm{~T}$ nominal value). This magnet was located on a vertical stage (AHT-65 PI MICOS, Eschbach, Germany); thereby, it was possible to change the applied magnetic field $\left(\mu_{0} H\right)$ to the microsystem by changing the relative distance. The deflection was captured with a CCD camera (Imaging Pixelfly, 205 X2 1475, Germany) in plane, orthogonal to the microsystem.

\section{Young's Modulus of M-SRE}

The M-SRE Young's modulus ( $Y_{\mathrm{M} \text {-SRE }}$ ) for different FF concentrations was calculated by experimentally recording the deflection of cantilevers under the action of the gravity by using a confocal microscope (PL $\mu 2300$ Optical Imaging Profiler, Sensofar, Spain) with software (PL $\mu$ Confocal Imaging Profiler, Sensofar, Spain).

\section{Transmittance in the VIS-NIR}

The spectral response as a function of the FF concentration was studied by coupling a broadband light source (HL-2000, Ocean Optics, USA) to a multimode fiber optic (Thorlabs, Dachau, Germany) with a core diameter of $125 \mu \mathrm{m}$. Light emerging from this fiber was orthogonally coupled to each slab sample (with an identical optical path of $250 \mu \mathrm{m}$ in all cases). A second fiber optics with a core diameter of $230 \mu \mathrm{m}$ was used to collect light emerging from the M-SRE, which was directly connected to a 
spectrometer (HR 4000, Ocean Optics, USA). Ten consecutive scans (each of which requiring an integration time of $100 \mathrm{~ms}$ ) were done for statistics. A non-doped slab sample was used as reference.

\section{AUTHOR CONTRIBUTIONS}

SP did the fabrication and most of the characterization. XM-B performed the structural analysis and helped with the biocompatibility tests. RR-R did the biocompatibility experiments. JS conducted all the magnetic characterization. JP analyzed and verified all the mechanical characterization. JB, $\mathrm{AL}$, and $\mathrm{VC}$

\section{REFERENCES}

Ahn, S. K., Kasi, R. M., Kim, S. C., Sharma, N., and Zhou, Y. X. (2008). Stimuliresponsive polymer gels. Soft Matter 4, 1151-1157. doi:10.1039/b714376a

Alves, N. M., Pashkuleva, I., Reis, R. L., and Mano, J. F. (2010). Controlling cell behavior through the design of polymer surfaces. Small 6, 2208-2220. doi:10.1002/smll.201000233

Balasoiu, M., Lebedev, V. T., Orlova, D. N., and Bica, I. (2011). Magnetic field and particle concentration competitive effects on ferrofluid based silicone elastomer microstructure. Crystallogr. Rep. 56, 1177-1180. doi:10.1134/ S1063774511070054

Bergstrom, J. S., and Boyce, M. C. (1999). Mechanical behavior of particle filled elastomers. Rubber Chem. Technol. 72, 633-656. doi:10.5254/1.3538823

Brau, F., Vandeparre, H., Sabbah, A., Poulard, C., Boudaoud, A., and Damman, P. (2011). Multiple-length-scale elastic instability mimics parametric resonance of nonlinear oscillators. Nat. Phys. 7, 56-60. doi:10.1038/nphys1806

Carrillo, F., Gupta, S., Balooch, M., Marshall, S. J., Marshall, G. W., Pruitt, L., et al. (2005). Nanoindentation of polydimethylsiloxane elastomers: effect of crosslinking, work of adhesion, and fluid environment on elastic modulus. J. Mater. Res. 20, 2820-2830. doi:10.1557/JMR.2005.0354

Cheng, Q. F., Duan, J. L., Zhang, Q., and Jiang, L. (2015). Learning from nature: constructing integrated graphene-based artificial nacre. ACS Nano 9, 2231-2234. doi:10.1021/acsnano.5b01126

Cullity, B. D. (1974). Introduction to Magnetic Materials. Reading, MA: Addison-Wesley.

Darder, M., Aranda, P., Burgos-Asperilla, L., Llobera, A., Cadarso, V. J., FernandezSanchez, C., et al. (2010). Algae-silica systems as functional hybrid materials. J. Mater. Chem. 20, 9362-9369. doi:10.1039/B913269D

Darder, M., Aranda, P., and Ruiz-Hitzky, E. (2007). Bionanocomposites: a new concept of ecological, bioinspired, and functional hybrid materials. Adv. Mater. Weinheim 19, 1309-1319. doi:10.1002/adma.200602328

de Pedro, S., Cadarso, V. J., Munoz-Berbel, X., Plaza, J. A., Sort, J., Brugger, J., et al. (2014). PDMS-based, magnetically actuated variable optical attenuators obtained by soft lithography and inkjet printing technologies. Sens. Actuators A Phys. 215, 30-35. doi:10.1016/j.sna.2014.01.021

Filipcsei, G., Csetneki, I., Szilagyi, A., and Zrinyi, M. (2007). "Magnetic field-responsive smart polymer composites," in Oligomers Polymer Composites Molecular Imprinting (Berlin: Springer-Verlag), 137-189.

Fisher, O. Z., Khademhosseini, A., Langer, R., and Peppas, N. A. (2010). Bioinspired materials for controlling stem cell fate. Acc. Chem. Res. 43, 419-428. doi:10.1021/ar900226q

Fuard, D., Tzvetkova-Chevolleau, T., Decossas, S., Tracqui, P., and Schiavone, P. (2008). Optimization of poly-di-methyl-siloxane (PDMS) substrates for studying cellular adhesion and motility. Microelectron. Eng. 85, 1289-1293. doi:10.1016/j.mee.2008.02.004

Giammanco, G. E., Sosnofsky, C. T., and Ostrowski, A. D. (2015). Light-responsive iron(III)-polysaccharide coordination hydrogels for controlled delivery. ACS Appl. Mater. Interfaces 7, 3068-3076. doi:10.1021/am506772x

Han, Y., Hong, W., and Faidley, L. A. E. (2013). Field-stiffening effect of magneto-rheological elastomers. Int. J. Solids Struct. 50, 2281-2288. doi:10.1016/j. ijsolstr.2013.03.030 designed the work, supervised the results, and monitored the writing.

\section{FUNDING}

The research leading to these results has received funding from the European Research Council under the European Community's Seventh Framework Programme (FP7/2007-2013) ERC grant agreement no. 209243. Partial financial support from the 2014SGR-1015 (DGU) is acknowledged. Dr. XM-B was supported by the "Ramón y Cajal" program from the Spanish Government. MINAHE4:TEC2011-29140-C03-01.

Hong, R. Y., Feng, B., Chen, L. L., Liu, G. H., Li, H. Z., Zheng, Y., et al. (2008). Synthesis, characterization and MRI application of dextran-coated $\mathrm{Fe}_{3} \mathrm{O}_{4}$ magnetic nanoparticles. Biochem. Eng. J. 42, 290-300. doi:10.1016/j.bej.2008.07.009

Hotz, N., Wilcke, L., and Weber, W. (2013). Design, synthesis, and application of stimulus-sensing biohybrid hydrogels. Macromol. Rapid Commun. 34 1594-1610. doi:10.1002/marc.201300468

Ibarlucea, B., Fernandez-Rosas, E., Vila-Planas, J., Demming, S., Nogues, C. Plaza, J. A., et al. (2010). Cell screening using disposable photonic lab on a chip systems. Anal. Chem. 82, 4246-4251. doi:10.1021/ac100590z

Ivaneyko, D., Toshchevikov, V., Saphiannikova, M., and Heinrich, G. (2012). Effects of particle distribution on mechanical properties of magneto-sensitive elastomers in a homogeneous magnetic field. Condens. Matter Phys. 15, 1-12. doi:10.5488/CMP.15.33601

Jeong, B., and Gutowska, A. (2002). Lessons from nature: stimuli-responsive polymers and their biomedical applications. Trends Biotechnol. 20, 305-311. doi:10.1016/S0167-7799(02)01962-5

Johnston, I. D., McCluskey, D. K., Tan, C. K. L., and Tracey, M. C. (2014). Mechanical characterization of bulk Sylgard 184 for microfluidics and microengineering. J. Micromech. Microeng. 24, 035017. doi:10.1088/0960-1317/24/3/035017

Jordan, A., Scholz, R., Wust, P., Fahling, H., Krause, J., Wlodarczyk, W., et al. (1997). Effects of magnetic fluid hyperthermia $(\mathrm{MFH})$ on $\mathrm{C} 3 \mathrm{H}$ mammary carcinoma in vivo. Int. J. Hyperthermia 13, 587-605. doi:10.3109/02656739709023559

Kaiser, R., and Miskolcz, G. (1970). Magnetic properties of stable dispersions of subdomain magnetite particles. J. Appl. Phys. 41, 1064. doi:10.1063/1.1658812

Khoo, M., and Liu, C. (2001). Micro magnetic silicone elastomer membrane actuator. Sens. Actuators A Phys. 89, 259-266. doi:10.1016/S0924-4247(00)00559-8

Lu, A. H., Salabas, E. L., and Schuth, F. (2007). Magnetic nanoparticles: synthesis, protection, functionalization, and application. Angew. Chem. Int. Ed. 46, 1222-1244. doi:10.1002/anie.200602866

Lubbe, A. S., Alexiou, C., and Bergemann, C. (2001). Clinical applications of magnetic drug targeting. J. Surg. Res. 95, 200-206. doi:10.1006/jsre.2000. 6030

Mosmann, T. (1983). Rapid colorimetric assay for cellular growth and survival application to proliferation and cyto-toxicity assays. J. Immunol. Methods 65, 55-63. doi:10.1016/0022-1759(83)90303-4

Munoz-Berbel, X., Rodriguez-Rodriguez, R., Vigues, N., Demming, S., Mas, J., Buttgenbach, S., et al. (2013). Monolithically integrated biophotonic lab-on-achip for cell culture and simultaneous $\mathrm{pH}$ monitoring. Lab. Chip 13, 4239-4247. doi:10.1039/c3lc50746g

Pankhurst, Q. A., Connolly, J., Jones, S. K., and Dobson, J. (2003). Applications of magnetic nanoparticles in biomedicine. J. Phys. D Appl. Phys. 36, R167-R181. doi:10.1088/0022-3727/36/13/201

Pileni, M. P. (2001). Magnetic fluids: fabrication, magnetic properties, and organization of nanocrystals. Adv. Funct. Mater. 11, 323-336. doi:10.1002/1616-3028(200110)11:5<323::AID-ADFM323>3.0.CO;2-J

Pirmoradi, F., Cheng, L. N., and Chiao, M. (2010). A magnetic poly(dimethylesiloxane) composite membrane incorporated with uniformly dispersed, coated iron oxide nanoparticles. J. Micromech. Microeng. 20, 015032. doi:10.1088/0960-1317/20/1/015032

Rodriguez-Rodriguez, R., Plaza, J. A., Matchkov, V., Simonsen, U., Herrera, M. D., Buttgenbach, S., et al. (2014). "Opto-mechanical microbridles for the 
determination of structural and functional properties of small resistance arteries," in 2014 IEEE 27th International Conference on Micro Electro Mechanical Systems (MEMS) (New Jersey: IEEE), 869-872.

Roy, D., Cambre, J. N., and Sumerlin, B. S. (2010). Future perspectives and recent advances in stimuli-responsive materials. Prog. Polym. Sci. 35, 278-301. doi:10.1016/j.progpolymsci.2009.10.008

Sagara, Y., and Kato, T. (2009). Mechanically induced luminescence changes in molecular assemblies. Nat. Chem. 1, 605-610. doi:10.1038/nchem.411

Schattling, P., Jochum, F. D., and Theato, P. (2014). Multi-stimuli responsive polymers - the all-in-one talents. Polymer Chem. 5, 25-36. doi:10.1039/ C3PY00880K

Seo, S., Lee, J., Kwon, M. S., Seo, D., and Kim, J. (2015). Stimuli-responsive matrix-assisted colorimetric water indicator of polydiacetylene nanofibers. ACS Appl. Mater. Interfaces 7, 20342-20348. doi:10.1021/acsami.5b06058

Shanmuganathan, K., Capadona, J. R., Rowan, S. J., and Weder, C. (2010). Stimuliresponsive mechanically adaptive polymer nanocomposites. ACS Appl. Mater. Interfaces 2, 165-174. doi:10.1021/am9006337

Shi, C. X., Guo, Z. Q., Yan, Y. L., Zhu, S. Q., Xie, Y. S., Zhao, Y. S., et al. (2013). Self-assembly solid-state enhanced red emission of quinolinemalononitrile: optical waveguides and stimuli response. ACS Appl. Mater. Interfaces 5, 192-198. doi:10.1021/am302466m

Stuart, M. A. C., Huck, W. T. S., Genzer, J., Muller, M., Ober, C., Stamm, M., et al. (2010). Emerging applications of stimuli-responsive polymer materials. Nat. Mater. 9, 101-113. doi:10.1038/nmat2614

Taleb, S., Darmanin, T., and Guittard, F. (2014). Elaboration of voltage and ion exchange stimuli-responsive conducting polymers with selective switchable liquid-repellency. ACS Appl. Mater. Interfaces 6, 7953-7960. doi:10.1021/ am501279h

Terwagne, D., Brojan, M., and Reis, P. M. (2014). Smart morphable surfaces for aerodynamic drag control. Adv. Mater. Weinheim 26, 6608-6611. doi:10.1002/ adma.201401403

Vila-Planas, J., Fernandez-Rosas, E., Ibarlucea, B., Demming, S., Nogues, C., Plaza, J. A., et al. (2011). Cell analysis using a multiple internal reflection photonic lab-on-a-chip. Nat. Protoc. 6, 1642-1655. doi:10.1038/nprot.2011.383
Vivero-Escoto, J. L., Slowing, I. I., Trewyn, B. G., and Lin, V. S. Y. (2010). Mesoporous silica nanoparticles for intracellular controlled drug delivery. Small 6, 1952-1967. doi:10.1002/smll.200901789

Wang, Y. C., Shim, M. S., Levinson, N. S., Sung, H. W., and Xia, Y. N. (2014). Stimuli-responsive materials for controlled release of theranostic agents. Adv. Funct. Mater. 24, 4206-4220. doi:10.1002/adfm.201400279

Wu, M. H., Huang, S. B., and Lee, G. B. (2010). Microfluidic cell culture systems for drug research. Lab. Chip 10, 939-956. doi:10.1039/b921695b

Xia, Y. N., and Whitesides, G. M. (1998). Soft lithography. Angew. Chem. Int. Ed. 37, 550-575. doi:10.1002/(SICI)1521-3773(19980316)37:5<550::AIDANIE550>3.0.CO;2-G

Xiong, Y., Yan, K., Bentley, W. E., Deng, H. B., Du, Y. M., Payne, G. F., et al. (2014). Compartmentalized multilayer hydrogel formation using a stimulus-responsive self-assembling polysaccharide. ACS Appl. Mater. Interfaces 6, 2948-2957. doi:10.1021/am405544r

Yang, H., Yuan, B., Zhang, X., and Scherman, O. A. (2014). Supramolecular chemistry at interfaces: host-guest interactions for fabricating multifunctional biointerfaces. Acc. Chem. Res. 47, 2106-2115. doi:10.1021/ar500105t

Zhang, J. L., Srivastava, R. S., and Misra, R. D. K. (2007). Core-shell magnetite nanoparticles surface encapsulated with smart stimuli-responsive polymer: synthesis, characterization, and LCST of viable drug-targeting delivery system. Langmuir 23, 6342-6351. doi:10.1021/la0636199

Conflict of Interest Statement: The authors declare that the research was conducted in the absence of any commercial or financial relationships that could be construed as a potential conflict of interest.

Copyright (c) 2016 de Pedro, Muñoz-Berbel, Rodríguez-Rodríguez, Sort, Plaza, Brugger, Llobera and Cadarso. This is an open-access article distributed under the terms of the Creative Commons Attribution License (CC BY). The use, distribution or reproduction in other forums is permitted, provided the original author(s) or licensor are credited and that the original publication in this journal is cited, in accordance with accepted academic practice. No use, distribution or reproduction is permitted which does not comply with these terms. 